\title{
Degradação do Corante Azul Reativo 5G Pelo \\ Processo Oxidativo Avançado UV/ $\mathrm{H}_{2} \mathrm{O}_{2}$
}

\section{e-Xactal}

ISSN: 1984-3151

\author{
Degradation of Reactive Blue 5G Dye by UV/ $\mathrm{H}_{2} \mathrm{O}_{2}$ Advanced \\ OXIDATION PROCESS
}

\author{
Denis Rafael de Souza Lima'; Isabela Luiza Alves de Almeida²; Vanderlei Inácio de Paula ${ }^{3}$ \\ 1 Graduando em Engenharia Química. Centro Universitário \\ Padre Anchieta - UniAnchieta, 2016. Jundiaí, SP. \\ denis rsl@yahoo.com.br. \\ 2 Graduanda em Engenharia Química. Centro Universitário \\ Padre Anchieta - UniAnchieta, 2016. Jundiaí, SP. \\ isabela poulain@yahoo.com.br \\ 3 Mestre em Química Inorgânica. Universidade Estadual de \\ Campinas, 2004. Professor do Centro Universitário Padre \\ Anchieta - UniAnchieta, Jundiaí, SP. \\ vanderleip@anchieta.br.
}

Recebido em: 12/08/2016 - Aprovado em: 02/11/2016 - Disponibilizado em: 30/11/2016

RESUMO: Neste trabalho avaliou-se a aplicação do processo oxidativo avançado (POA) de foto-peroxidação $\left(\mathrm{UV} / \mathrm{H}_{2} \mathrm{O}_{2}\right)$ na degradação do azocorante têxtil comercial Azul Reativo 5G. Foram preparadas soluções sintéticas contendo o corante e tratadas através do respectivo POA, sendo a eficiência do tratamento avaliada através dos seguintes parâmetros: descoloração, redução da demanda química de oxigênio (DQO) e ecotoxicidade com sementes de Lactuca sativa. Após 30 minutos de tratamento em condições otimizadas na presença de peróxido de hidrogênio e radiação ultravioleta, foi observada descoloração na ordem de $91,8 \%$ sem redução significativa da DQO $(1,8 \%)$, além de diminuição da ecotoxicidade da solução, indicando a viabilidade da aplicação do processo $U V / \mathrm{H}_{2} \mathrm{O}_{2}$ para tratamento de resíduos líquidos contendo este azocorante.

PalavRas-CHAVE: Processos Oxidativos Avançados. UV/ $\mathrm{H}_{2} \mathrm{O}_{2}$. Degradação. Corante Têxtil.

ABSTRACT: In the present paper was evaluated the application of advanced oxidation process (AOP) photoperoxidation $\left(\mathrm{UV} / \mathrm{H}_{2} \mathrm{O}_{2}\right)$ applied to degradation of the commercial textile azo dye Reactive Blue 5G. Synthetic solutions were prepared containing the dye, treated through the respective AOP and the efficiency of the treatment evaluated by the following parameters: discoloration, reduction of chemical oxygen demand (COD) and ecotoxicity with Lactuca sativa seeds. After 30 minutes of treatment in optimized conditions in the presence of hydrogen peroxide and ultraviolet radiation, discoloration was observed in order of $91.8 \%$ without significant reduction of $C O D(1.8 \%)$, but decrease in the ecotoxicity of the solution, indicating feasibility of the process $U \mathrm{~V} / \mathrm{H}_{2} \mathrm{O}_{2}$ for treating liquid wastes containing this azo dye.

KEYWORDS: Advanced Oxidation Processes. $U \mathrm{~V} / \mathrm{H}_{2} \mathrm{O}_{2}$. Degradation. Textile Dye.

\section{INTRODUÇÃo}

A poluição dos recursos hídricos por efluentes industriais contendo compostos orgânicos e substâncias tóxicas é um assunto de crescente interesse devido à preocupação com as questões ambientais e aumentos no rigor da legislação nos últimos anos (ARAÚJO et al., 2016). 
Nesse contexto, a indústria têxtil é uma das maiores geradoras de efluentes líquidos, dentre diversas tipologias industriais, com consumo estimado de 150 litros de água para produção de um quilo de tecido, sendo $88 \%$ desse volume descartado como efluente líquido e os $12 \%$ restantes perdidos por evaporação (LEÃO et al., 2002). Além de corantes, pigmentos e produtos auxiliares, os efluentes têxteis possuem grandes quantidades de compostos orgânicos e inorgânicos que, isolados ou associados entre si, apresentam toxicidade à vida aquática, diminuindo também o oxigênio dissolvido e modificando as propriedades e características físicas e químicas dos corpos hídricos se descartados sem tratamento adequado (CISNEROS; ESPINOZA; LITTER, 2002).

Atualmente, uns dos principais tipos de corantes utilizados pelas indústrias e lavanderias têxteis são os corantes reativos, normalmente aplicados para tingimento de tecidos tipo jeans, pois obtêm tintos com brilho excepcional, boa igualação e excelente solidez (CERVELIN, 2010). Entretanto, estudos têm mostrado que esses compostos e seus subprodutos podem ser carcinogênicos e/ou mutagênicos (KUNZ; PERALTAZAMORA, 2002), necessitando de processos mais eficientes para 0 tratamento de seus efluentes, capazes de degradar e mineralizar esses compostos recalcitrantes.

Devido à estrutura química complexa dos corantes presentes nos efluentes da indústria têxtil, os sistemas convencionais de tratamento (coagulação/floculação, adsorção com carvão ativo, precipitação, degradação biológica) nem sempre são viáveis para o seu tratamento, pois possuem limitações termodinâmicas e cinéticas ao atacar compostos refratários (CISNEROS; ESPINOZA; LITTER, 2002), além de muitas vezes apenas transferir os poluentes de fase, gerando resíduos sólidos que também deverão ser tratados posteriormente.
Nas últimas décadas os Processos Oxidativos Avançados (POAs) têm se destacado na pesquisa e desenvolvimento de tecnologias de tratamento de águas residuais, por se tratar de métodos eficientes que reduzem os impactos ambientais. A eficácia dos POAs depende da geração de radicais livres reativos, sendo o mais importante o radical hidroxila ( $\mathrm{HO \bullet}$ ), capazes de oxidar a maioria das moléculas orgânicas recalcitrantes de forma não seletiva (ARAÚJO et al., 2016). Dentre os POAs, o processo que combina peróxido de hidrogênio e radiação ultravioleta tem se destacado de forma bem-sucedida no tratamento de diversos poluentes, como por exemplo: pesticidas e herbicidas (OLIVEIRA et al., 2014; ROZAS et al., 2016), fármacos (NAPOLEÃO et al., 2015), corantes têxteis (RACYTE; RIMEIKA, 2008), entre outros.

Dentre os diversos processos classificados como POAs, a combinação de luz ultravioleta e peróxido de hidrogênio $\left(\mathrm{UV} / \mathrm{H}_{2} \mathrm{O}_{2}\right)$ é o mais antigo (LITTER, 2005), ocorrendo em duas etapas principais: formação de radicais hidroxila pela fotólise do peróxido de hidrogênio (Equação 1) e oxidação das moléculas orgânicas pelos radicais formados (Equações 2 a 4) (WILL et al., 2004). Em certos casos, a energia do fóton também é capaz de romper ligações químicas nas moléculas orgânicas, promovendo sua degradação a compostos mais simples, como $\mathrm{CO}_{2}$, $\mathrm{H}_{2} \mathrm{O}$ e ânions inorgânicos (SUBTIL; MIERZWA; HESPANHOL, 2009).

$$
\begin{aligned}
& \mathrm{H}_{2} \mathrm{O}_{2}+h v \rightarrow 2 \cdot \mathrm{OH} \\
& \mathrm{OH}+\mathrm{RH} \rightarrow \mathrm{H}_{2} \mathrm{O}+\mathrm{R} . \\
& \mathrm{OH}+\mathrm{X}_{2} \mathrm{C}=\mathrm{CX}_{2} \rightarrow \mathrm{X}_{2} \mathrm{C}(\mathrm{OH})-\mathrm{CX}_{2} \\
& \mathrm{OH}+\mathrm{RX} \rightarrow \mathrm{OH}^{-}+\mathrm{XR}^{+}
\end{aligned}
$$

Desse modo, esse trabalho objetivou avaliar a eficiência/viabilidade da degradação de um efluente sintético contendo o azocorante têxtil Azul Reativo 5G (AR5G) pelo processo oxidativo $\mathrm{UV} / \mathrm{H}_{2} \mathrm{O}_{2}$, através de 
análises de descoloração, demanda química de oxigênio (DQO) e ecotoxicidade, parâmetros de interesse ambiental e que são comuns em efluentes reais da indústria têxtil.

\section{MATERIAIS e MÉtodos}

\subsection{Preparação da Amostra e Reagentes}

A matriz estudada foi o corante têxtil comercial Azul Reativo 5G, fornecido pela Texpal Química Ltda (Figura 1). Sua estrutura possui o grupo cromóforo azo, responsável pela cor, sulfonato, responsável pela solubilidade e caráter aniônico e os grupamentos reativos vinilsulfona e monoclorotriazina. Pertence à classe de corantes reativos bifuncionais (KOPRIVANAC et al., 2005).

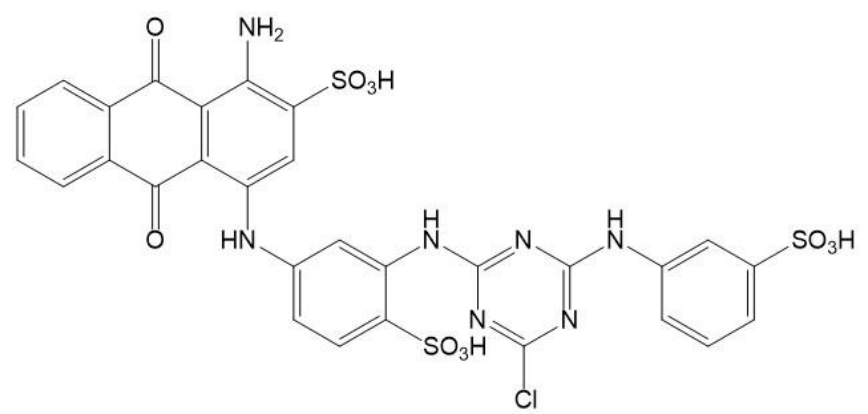

Figura 1 - Estrutura química do corante azul reativo $5 \mathrm{G}$.

Fonte - FIORENTIN et al., 2010.

Para os ensaios de degradação foram preparadas soluções aquosas na concentração de $100 \mathrm{mg} \cdot \mathrm{L}^{-1}$ do corante, utilizando água destilada.

Também foram utilizadas soluções comerciais padronizadas de peróxido de hidrogênio $30 \% \mathrm{v} / \mathrm{v}$ (Synth) e soluções estoque de $\mathrm{NaOH}$ e $\mathrm{HCl}$ (Vetec) para ajuste de $\mathrm{pH}$.

\subsection{Ensaios de Degradação}

Os ensaios de degradação foram realizados utilizando um reator fotoquímico de bancada construído anteriormente (LIMA; PAULA, 2014), representado na
Figura 2. A fonte de radiação empregada foi uma lâmpada de vapor de mercúrio de alta pressão, 250W, marca Empalux, cujo envoltório externo foi previamente removido. Esse tipo de lâmpada apresenta como característica elevado fluxo radiante e várias bandas alargadas na região do espectro UV, sendo os picos em 365, 436 e $446 \mathrm{~nm}$ os mais intensos, ocorrendo também emissão não desprezível no visível (CAVICCHIOLI; GUTZ, 2003).

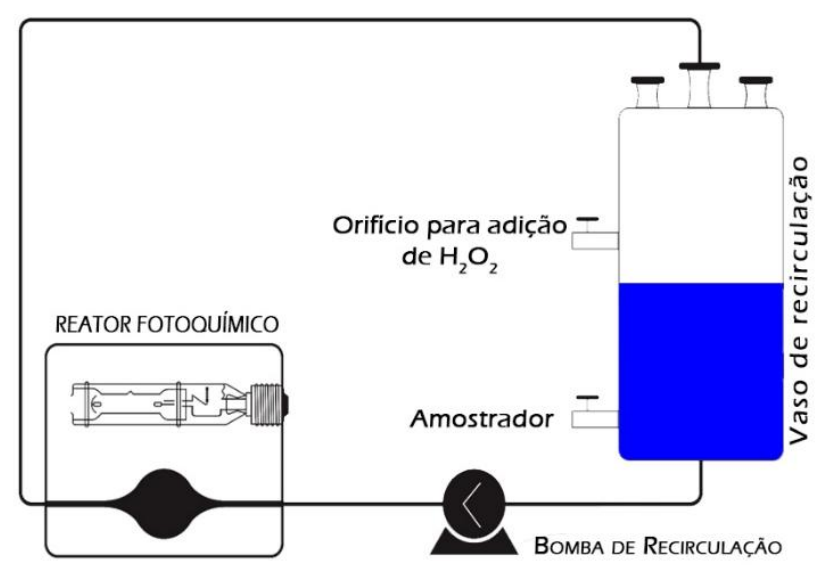

Figura 2 - Esquema do reator fotoquímico utilizado. Fonte - LIMA; PAULA, 2014.

O volume de solução irradiado foi de $1,0 \mathrm{~L}$ com fluxo de recirculação de 0,35 L.min ${ }^{-1} \cdot \mathrm{O}$ pH inicial da solução foi ajustado em 3,8 $\pm 0,2$ e a temperatura de operação $30 \pm 3^{\circ} \mathrm{C}$, com posterior adição do peróxido de hidrogênio. Foram retiradas alíquotas para testes no início da degradação e em intervalos de 10 minutos, até o período final de 30 minutos.

A descoloração foi acompanhada pela obtenção dos espectros de absorção UV-Vis na faixa de 250 a 750 $\mathrm{nm}$, utilizando espectrofotômetro SmartSpectro (Lamotte), no comprimento de onda de máxima absorbância do AR5G determinado experimentalmente, $620 \mathrm{~nm}$.

A redução da demanda química de oxigênio (DQO) foi determinada nas amostras pré e pós-tratamento do efluente sintético através do método colorimétrico (SM 5220D), conforme Standard Methods for the 
Examination of Water and Wastewater, 22nd edition (APHA, 2012).

\subsection{ENSAIOS DE ECOTOXICIDADE}

Os testes de toxidade foram realizados com sementes de alface (Lactuca sativa), de acordo com a metodologia adaptada de Sobrero e Ronco (2004). As soluções de AR5G bruto e tratado sofreram diluições com adição de água destilada, nos seguintes percentuais: $100 \%$ da solução, $80 \%, 50 \%, 30 \%$ e $10 \%$ $\mathrm{v} / \mathrm{v}$.

De acordo com o protocolo de ensaio, as sementes foram selecionadas e acondicionadas em placas de Petri de $9 \mathrm{~cm}$ de diâmetro previamente identificadas e preenchidas com papel filtro qualitativo de tamanho apropriado, sendo umedecidas com $4 \mathrm{~mL}$ de amostra, em triplicata. A amostra controle foi saturada apenas com água destilada. Após a preparação, as placas foram cobertas para evitar perda de umidade e incubadas ao abrigo da luz pelo período de $120 \mathrm{~h}$ em ambiente climatizado $\left(22 \pm 2^{\circ} \mathrm{C}\right)$.

Ao término da incubação, a avaliação dos efeitos foi realizada em comparação à amostra de controle, sendo quantificados os efeitos na germinação e no crescimento das raízes e hipocótilos das plântulas de alface (Figura 3).

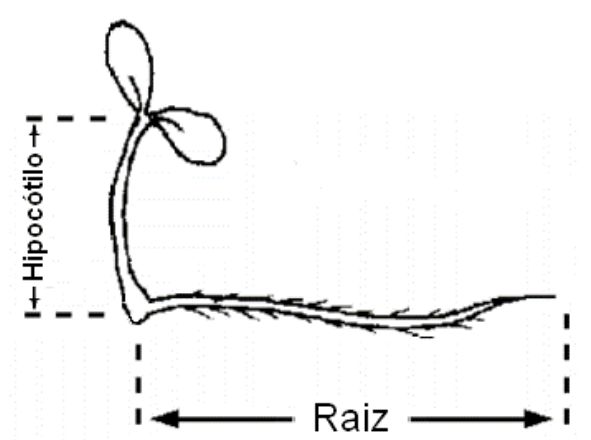

Figura 3 - Morfologia da plântula de Lactuca sativa. Fonte - Adaptado de CORNELL UNIVERSITY AND PENN STATE UNIVERSITY, 2009.

O delineamento experimental aplicado aos testes de toxidade foi inteiramente casualizado e os dados submetidos à análise de variância, sendo as médias de inibição da germinação e crescimento comparadas pelo teste de Scott-Knott ao nível de $5 \%$ de probabilidade, utilizando 0 software estatístico ASSISTAT 7.7.

A taxa de germinação relativa (\%GR) para cada diluição foi calculada através da Equação 5.

$$
\% G R=\frac{\text { № SGA }}{\text { № SGC }} * 100
$$

Em que, №SGA é o número de sementes germinadas na amostra e №SGC é o número de sementes germinadas no controle.

Os percentuais de inibição do crescimento relativo das raízes (\%ICRRz), hipocótilos (\%ICRHp) e total (\%ICTotal) foram calculados a partir das médias obtidas para cada diluição, através das Equações 6 a 8.

$$
\begin{aligned}
& \% I C R R z=\frac{M C R z C-M C R z A}{M C R z C} * 100 \\
& \% I C R H p=\frac{M C H p C-M C H p A}{M C H p C} * 100 \\
& \% I C T o t a l=\frac{\text { MCTotalC }- \text { MCTotalA }}{\text { MCTotalC }} * 100
\end{aligned}
$$

Nas quais:

MCRzA = média de crescimento das raízes na amostra.

$\mathrm{MCRzC}=$ média de crescimento das raízes no controle.

$\mathrm{MCHpA}=$ média do crescimento dos hipocótilos na amostra.

$\mathrm{MCHpC}=$ média de crescimento dos hipocótilos no controle.

MCTotal $\mathrm{A}=$ média de crescimento total na amostra .

MCTotalC = média de crescimento total no controle.

\section{Resultados e Discussão}

A concentração ideal de peróxido de hidrogênio foi avaliada para a descoloração das soluções de AR5G, conforme Figura 4. 


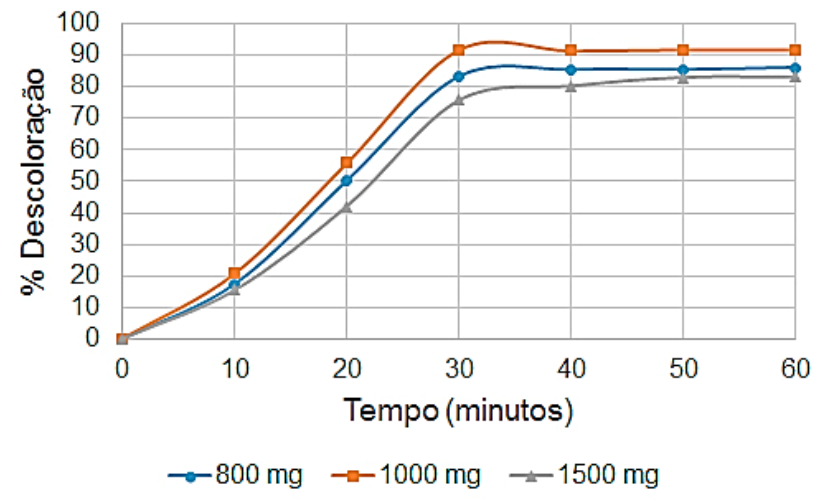

Figura 4 - Descoloração da solução de AR5G em função da concentração de $\mathrm{H}_{2} \mathrm{O}_{2}$.

$[A R 5 G]_{\text {inicial }}=100 \mathrm{mg}^{-L^{-1}} ; \mathrm{V}=1 \mathrm{~L} ; \mathrm{pH}$ inicial $=3,8 ;$ Vazão $=0,35$ L.min ${ }^{-1} ; \mathrm{T}=30 \pm 3^{\circ} \mathrm{C}$.

Fonte - Próprio autor.

Pode-se observar que nos ensaios realizados, o maior percentual de descoloração $(91,8 \%)$ após 60 minutos de reação foi obtido utilizando a concentração de 1000 mg. $\mathrm{L}^{-1}$ de $\mathrm{H}_{2} \mathrm{O}_{2}$, uma vez que as concentrações de 800 e 1500 mg obtiveram descoloração de 86,1 e $83,0 \%$, respectivamente.

Nota-se que o excesso de peróxido provoca uma diminuição na descoloração, o que pode ocorrer devido às reações competitivas de efeito inibitório para a degradação, pois os radicais hidroxila $(\mathrm{HO} \bullet)$ tendem a se recombinar e reagir conforme as Equações 9 a 11, formando radicais hidroperoxila $\left(\mathrm{HO}_{2} \bullet\right)$ que prejudicam o processo de degradação por serem menos reativos (NOGUEIRA et al., 2007).

$$
\begin{aligned}
& \mathrm{HO} \bullet+\mathrm{H}_{2} \mathrm{O}_{2} \rightarrow \mathrm{H}_{2} \mathrm{O}+\mathrm{HO}_{2} \bullet \\
& \mathrm{HO}_{2} \bullet+\mathrm{HO} \bullet \rightarrow \mathrm{H}_{2} \mathrm{O}+\mathrm{O}_{2} \\
& 2 \mathrm{HO}_{2} \bullet \rightarrow \mathrm{H}_{2} \mathrm{O}_{2}+\mathrm{O}_{2}
\end{aligned}
$$

Determinada a concentração ideal de oxidante, 1000 $\mathrm{mg} . \mathrm{L}^{-1}$, foram realizados experimentos individuais com apenas a fotólise do corante AR5G e adição de $\mathrm{H}_{2} \mathrm{O}_{2}$ na ausência de luz, porém nenhuma dessas condições foi capaz de descolorir satisfatoriamente o composto (resultados < 5\% remoção), demonstrando sua fotoestabilidade e comprovando a sinergia da combinação $\mathrm{UV} / \mathrm{H}_{2} \mathrm{O}_{2}$ para degradação do mesmo.

A Figura 5 apresenta o comportamento espectral do corante quando tratado via processo $\mathrm{UV} / \mathrm{H}_{2} \mathrm{O}_{2}$ pelo período de 30 minutos de irradiação.

Pode-se observar a descoloração da amostra pela diminuição do pico de $620 \mathrm{~nm}$, característico da ligação azo $(\mathrm{N}=\mathrm{N})$ presente na molécula. Esse tipo de ligação apresenta pares de elétrons livres que, ao se ligar ao radical hidroxila, possibilitam sua quebra e consequentemente a remoção da cor (SOUZA et al., 2016). Na região UV do espectro também houve diminuição das absorbâncias, indicando uma diminuição da aromaticidade.

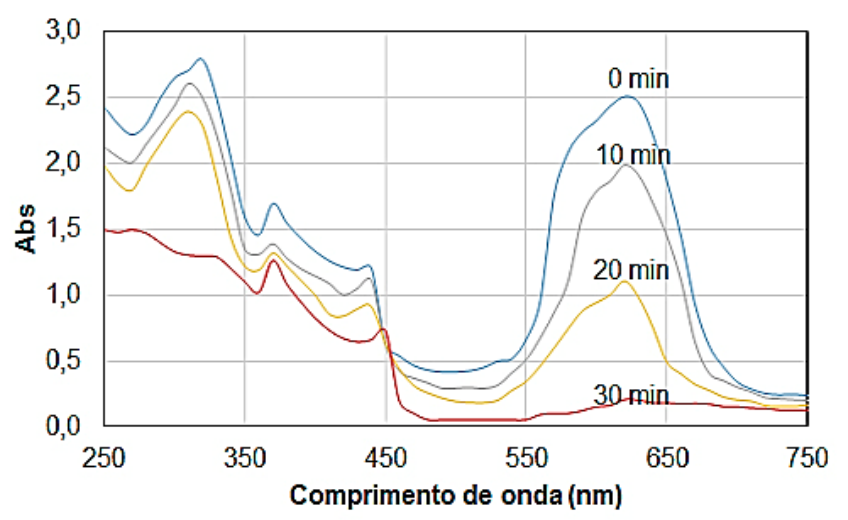

Figura 5 - Espectros de absorção do corante AR5G em diferentes tempos de tratamento.

$[A R 5 G]_{\text {inicial }}=100 \mathrm{mg}^{-L^{-1}} ; \mathrm{V}=1 \mathrm{~L} ; \mathrm{pH}$ inicial $=3,8 ;$ Vazão $=0,35 \mathrm{~L} \cdot \mathrm{min}^{-1} ; \mathrm{T}=30 \pm 3^{\circ} \mathrm{C}$. Fonte - Próprio autor.

No comprimento de onda de maior absorção do efluente, $620 \mathrm{~nm}$, mais de $90 \%$ de descoloração foi alcançada em 30 minutos de irradiação, estabilizando após esse período. A Figura 6 apresenta o aspecto visual antes e após o tratamento. 


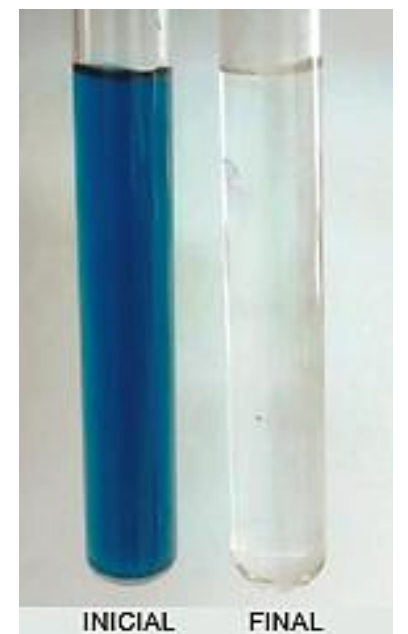

Figura 6 - Amostras de AR5G antes e após o tratamento pelo $\mathrm{POA} U \mathrm{UV} / \mathrm{H}_{2} \mathrm{O}_{2}$.

Fonte - Próprio autor.

A Tabela 1 apresenta os resultados das análises de demanda química de oxigênio para as amostras de AR5G bruto e tratado.

\section{Tabela 1}

Dados de DQO para (ES) bruto e tratado

\begin{tabular}{c|c}
\hline Amostra & DQO $\left(\mathrm{mg} \mathrm{O}_{2} / \mathrm{L}\right)$ \\
\hline ES Bruto & 112 \\
ES Tratado & 110 \\
\hline
\end{tabular}

Fonte - Próprio autor.
De acordo com a Tabela 1, o percentual de eficiência na redução da $\mathrm{DQO}$ obtido não foi significativo, apenas 1,8\%, o que pode ser explicado pelo fato de que durante a descoloração, novas substâncias orgânicas podem ter sido formadas, as quais apesar de não serem coloridas, ainda necessitam de maiores tempos para sua efetiva mineralização à $\mathrm{CO}_{2}, \mathrm{H}_{2} \mathrm{O} \mathrm{e}$ sais inorgânicos (KURBUS; LE MARECHAL; VONCINA, 2003).

Os ensaios de ecotoxicidade foram realizados com as soluções de AR5G bruto e após 30 minutos de tratamento. A espécie Lactuca sativa foi capaz de se estabelecer em meio potencialmente tóxico e a se desenvolver parcialmente, apresentando efeitos letais (inibição da germinação) e subletais (inibição do crescimento das raízes e hipocótilos).

A Tabela 2 apresenta os valores médios obtidos para a germinação e crescimento das plântulas de alface expostas às amostras em diferentes proporções. Como controle positivo foi utilizado apenas água destilada.

Tabela 2

Resultados para os ensaios de germinação e comprimento da raiz, hipocótilo e total das plântulas de alface ( $L$. sativa) submetidas ao efluente bruto e tratado.

\begin{tabular}{c|c|c|c|c}
\hline Tratamento & Germinação $(\%)$ & $\begin{array}{c}\text { Comprimento da raiz } \\
(\mathrm{mm})\end{array}$ & $\begin{array}{c}\text { Comprimento do } \\
\text { hipocótilo }(\mathrm{mm})\end{array}$ & $\begin{array}{c}\text { Comprimento total } \\
(\mathrm{mm})\end{array}$ \\
\hline Controle & $100,0 \mathrm{a}$ & $18,1 \pm 0,9 \mathrm{a}$ & $15,4 \pm 1,3 \mathrm{a}$ & $33,5 \pm 1,5 \mathrm{a}$ \\
EB 100\% & $83,3 \mathrm{~b}$ & $7,7 \pm 1,0 \mathrm{c}$ & $9,9 \pm 1,2 \mathrm{~b}$ & $17,6 \pm 1,5 \mathrm{c}$ \\
EB 80\% & $80,0 \mathrm{~b}$ & $9,9 \pm 1,9 \mathrm{c}$ & $11,2 \pm 0,6 \mathrm{~b}$ & $21,1 \pm 2,0 \mathrm{c}$ \\
EB 50\% & $90,0 \mathrm{~b}$ & $12,9 \pm 0,4 \mathrm{~b}$ & $11,3 \pm 1,2 \mathrm{~b}$ & $24,3 \pm 1,0 \mathrm{~b}$ \\
EB 30\% & $93,3 \mathrm{a}$ & $14,5 \pm 0,8 \mathrm{a}$ & $12,4 \pm 1,3 \mathrm{~b}$ & $26,9 \pm 1,1 \mathrm{~b}$ \\
EB 10\% & $100,0 \mathrm{a}$ & $18,5 \pm 1,4 \mathrm{a}$ & $14,7 \pm 1,3 \mathrm{a}$ & $33,3 \pm 1,5 \mathrm{a}$ \\
ET 100\% & $100,0 \mathrm{a}$ & $16,9 \pm 2,0 \mathrm{a}$ & $14,6 \pm 1,4 \mathrm{a}$ & $31,6 \pm 2,2 \mathrm{a}$ \\
ET 80\% & $100,0 \mathrm{a}$ & $16,2 \pm 1,4 \mathrm{a}$ & $15,8 \pm 1,0 \mathrm{a}$ & $32,0 \pm 1,8 \mathrm{a}$ \\
ET 50\% & $100,0 \mathrm{a}$ & $19,4 \pm 1,9 \mathrm{a}$ & $16,3 \pm 0,9 \mathrm{a}$ & $35,7 \pm 2,0 \mathrm{a}$ \\
ET 30\% & $100,0 \mathrm{a}$ & $16,8 \pm 2,0 \mathrm{a}$ & $16,5 \pm 1,3 \mathrm{a}$ & $33,3 \pm 2,1 \mathrm{a}$ \\
ET 10\% & $100,0 \mathrm{a}$ & $19,5 \pm 1,8 \mathrm{a}$ & $16,0 \pm 0,9 \mathrm{a}$ & $35,5 \pm 2,0 \mathrm{a}$ \\
\hline
\end{tabular}

$\mathrm{EB}=$ Efluente Bruto / ET = Efluente Tratado. Médias seguidas pela mesma letra, na mesma coluna, não diferem entre si pelo teste de Scott Knott ao nível de $5 \%$ de probabilidade. Média \pm erro.

Fonte - Próprio autor. 
O corante AR5G sem tratamento se mostrou inibidor ao analisar sua germinação, comprimento das raízes, radículas e total. Comparando com as amostras expostas ao controle, houve diferença significativa no percentual de germinação das amostras contendo 100,80 e $50 \%$ do corante bruto, apresentando germinação relativa de $83.3 \%, 80.0 \%$ e $90.0 \%$, respectivamente. Todas as amostras tratadas apresentaram 100\% de germinação (Figura 7), indicando que 0 processo $\mathrm{UV} / \mathrm{H}_{2} \mathrm{O}_{2}$ produziu intermediários responsáveis pela diminuição da toxicidade, sendo que, quanto maior a diluição, menor o efeito de letalidade frente aos organismos-teste.

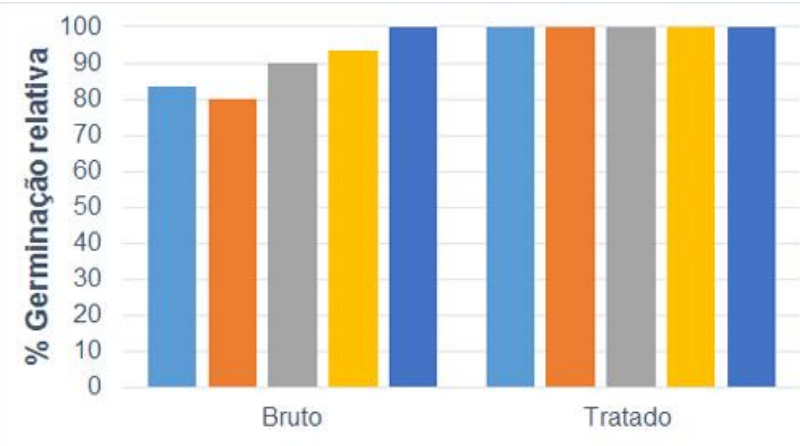

$=100 \%$ de $\mathrm{ES} \| 80 \%$ de $\mathrm{ES}=50 \%$ de $\mathrm{ES}=30 \%$ de $\mathrm{ES} \| 10 \%$ de $\mathrm{ES}$

Figura 7 - Germinação Relativa ao controle para o ES contendo AR5G bruto e tratado.

Fonte - Próprio autor.

Nas amostras germinadas foi possível calcular a inibição relativa ao crescimento das raízes, hipocótilos e total. Os resultados podem ser observados nas Figuras 8 a 10, respectivamente.

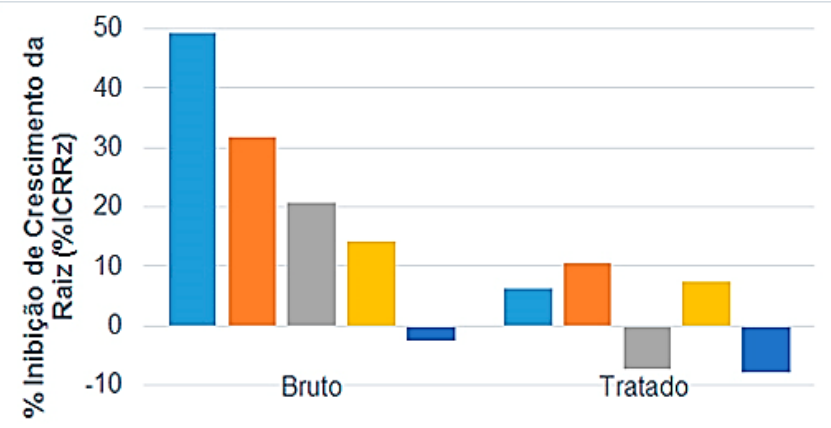

$\square 100 \%$ de ES $\square 80 \%$ de ES $\square 50 \%$ de ES $\square 30 \%$ de ES $\square 10 \%$ de ES

Figura 8 - Inibição de Crescimento da Raiz (\%ICRRz) relativo ao controle para o Efluente Sintético (ES) contendo AR5G bruto e tratado.

Fonte - Próprio autor.

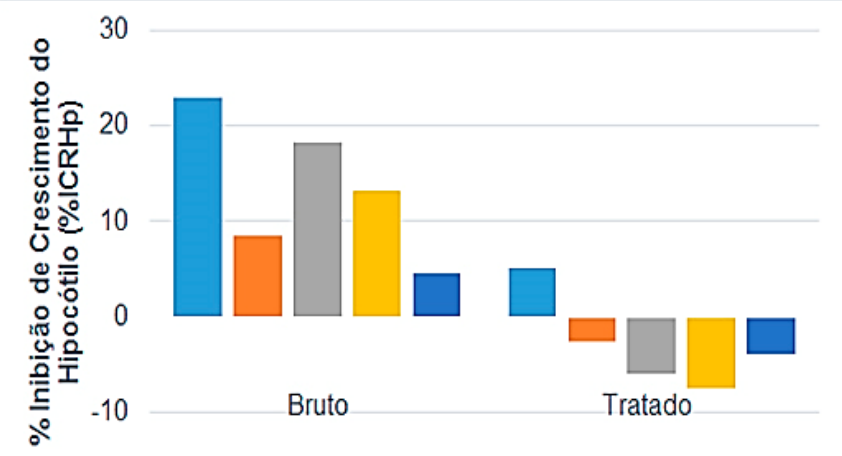

$\square 100 \%$ de ES $\square 80 \%$ de ES $\square 50 \%$ de ES $\square 30 \%$ de ES $\square 10 \%$ de ES

Figura 9 - Inibição de Crescimento do Hipocótilo (\%ICRHp) relativo ao controle para o Efluente Sintético (ES) contendo AR5G bruto e tratado. Fonte - Próprio autor.

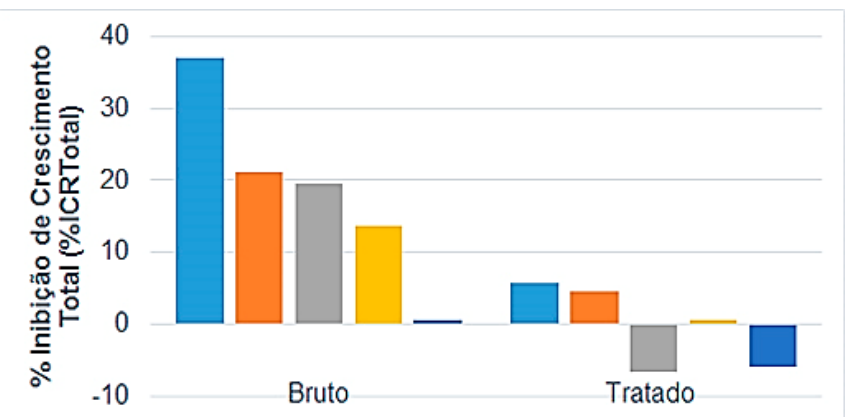

घ $100 \%$ de ES $\square 80 \%$ de ES $\square 50 \%$ de ES $\backsim 30 \%$ de ES $\square 10 \%$ de ES

Figura 10 - Inibição de Crescimento Total (\%ICR $\left.{ }_{\text {Total }}\right)$ relativo ao controle para o Efluente Sintético (ES) contendo AR5G bruto e tratado. Fonte - Próprio autor.

De acordo com os resultados apresentados nas Figuras 8 a 10, pode-se observar redução da inibição do crescimento das plântulas de L. sativa no efluente tratado em todas as suas diluições, chegando a ocorrer inibições negativas em algumas amostras, devido ao desenvolvimento das raízes e/ou hipocótilos maior nas amostras do que no controle.

Os resultados demonstram que após o tratamento pelo processo oxidativo avançado $\mathrm{UV} / \mathrm{H}_{2} \mathrm{O}_{2}$ o corante AR5G teve seus efeitos fitotóxicos reduzidos significativamente. 


\section{CONCLUSÕES}

Os resultados obtidos neste trabalho constataram a viabilidade do processo $\mathrm{UV} / \mathrm{H}_{2} \mathrm{O}_{2}$ na degradação de efluentes têxteis contendo o azocorante azul reativo $5 G$.

Após 30 minutos de tratamento foram atingidos $91,8 \%$ de remoção de cor e 1,8\% de redução da DQO. Apesar da baixa redução da carga orgânica das amostras, dados da literatura indicam que tempos maiores de tratamento por POAs são capazes de atingir melhores resultados, pois geralmente a mineralização se inicia somente quando a descoloração se encontra praticamente completa (KURBUS; LE MARECHAL; VONCINA, 2003).
O teste de ecotoxidade utilizando Lactuca sativa evidenciou que o tratamento aplicado foi capaz de reduzir significativamente seus níveis fitotóxicos, sendo maior no efluente bruto que no tratado, devido à geração de intermediários menos nocivos ao meio ambiente.

\section{AGRADECIMENTOS}

Os autores agradecem programa institucional de pesquisa e iniciação científica do Centro Universitário Padre Anchieta (UniAnchieta) pela bolsa concedida.

Agradecem também a empresa Texpal Química Ltda., pelas amostras de corante fornecidas.

\section{REFERÊNCIAS}

APHA - AMERICAN PUBLIC HEALTH ASSOCIATION. Standard Methods for the Examination of Water and Wastewater, 22nd ed. Washington, DC: American Public Health Association, 2012.

ARAÚJO, K. S. de et al. Processos oxidativos avançados: uma revisão de fundamentos e aplicações no tratamento de águas residuais urbanas e efluentes industriais. Rev. Ambient. Água, Taubaté, v. 11, n. 2, p. 387-401, 2016.

CAVICCHIOLI, A.; GUTZ, I. G. R.; O uso de radiação ultravioleta para o pré-tratamento de amostras em análise inorgânica. Química Nova, v. 26, p. 913 - 921, 2003.

CERVELIN, P. C. Avaliação da remoção do corante comercial reativo azul 5G utilizando macrófita Salvínia sp. Dissertação, Programa de PósGraduação em Engenharia Química, Universidade do Oeste do Paraná, 2010.

CISNEROS, R. L.; ESPINOZA, A. G.; LITTER, M. I. Photodegradation of na azo dye of the textile industry. Chemosphere, v. 48, p. 393-399, 2002.

CORNELL UNIVERSITY AND PENN STATE UNIVERSITY. Environmental Inquiry, 2009. Disponível em:

<http://ei.cornell.edu/toxicology/bioassays/lettuce/data. html>. Acesso em: 8 ago. 2016.
FIORENTIN, L. D. et al. Biosorption of reactive blue $5 G$ dye onto drying orange bagasse in batch system: kinetic and equilibrium modeling, Chem. Eng. J. v. 163, p. $68-77,2010$.

KOPRIVANAC, $\mathrm{N}$. et al. Influence of iron on degradation of organic dyes in corona. Journal of Hazardous Materials. v. 117, p. 113-119, 2005.

KUNZ, A.; PERALTA-ZAMORA, P. Novas técnicas no tratamento de efluentes têxteis. Química Nova, São Paulo, v. 25, n. 1, p. 78-82, 2002.

KURBUS, T.; LE MARECHAL, A. M.; VONCINA, D. B. Comparison of $\mathrm{H}_{2} \mathrm{O}_{2} / \mathrm{UV}, \mathrm{H}_{2} \mathrm{O}_{2} / \mathrm{O}_{3}$ and $\mathrm{H}_{2} \mathrm{O}_{2} / \mathrm{Fe}^{2+}$ processes for the decolorisation of vinylsulphone reactive dyes. Dyes Pigm., v. 58, p. 245 -252, 2003.

LEÃO, M. D. et al. Controle Ambiental na Indústria Têxtil: Acabamento de Malhas. $1^{\underline{a}}$ ed. Belo Horizonte: Projeto Minas Ambiente, Editora Segrac, 2002.

LIMA, D. R. S.; PAULA, V. I. Construção de um Reator Fotoquímico em Fluxo e Avaliação da Degradação do Corante Azul Indigotina (FD\&C Blue No. 2). In: XXVIII Encontro Regional da Sociedade Brasileira de Química, 2014, Poços de Caldas. Anais do XXVIII Encontro Regional da Sociedade Brasileira de Química, 2014.

LITTER, M. I. Introduction to Photochemical Advanced Oxidation Processes for Water Treatment. Hdb. Env. Chem., v.2, p. 325-366, 2005. 
NAPOLEÃO, D. C. et al. Degradação do contaminante emergente paracetamol empregando processos oxidativos avançados. Revista Eletrônica em Gestão, Educação e Tecnologia Ambiental, v. 19, p. 1, 2015.

NOGUEIRA, R. F. P. et al. Fundamentos e aplicações ambientais dos processos Fenton e Foto-Fenton. Química Nova, v. 30, n. 2, p. 400-408, 2007.

OLIVEIRA, A. G. et al. Nascimento, R. F. Degradation of the pesticide chlorpyrifos in aqueous solutions with $\mathrm{UV} / \mathrm{H}_{2} \mathrm{O}_{2}$ : Optimization and effect of interfering anions. Journal of AOTs. Advanced Oxidation Technologies, v. 17, p. 133-138, 2014.

RACYTE, J.; RIMEIKA, M. UV/ $\mathrm{H}_{2} \mathrm{O}_{2}$-Advanced Oxidation processes possibilities for textile reactive dyes decolorization. Lithuania: The $7^{\text {th }}$ int. Conference Faculty of Environmental Eng., Vilnius Gediminas Technical Univ., may 22-23, p. 684-689, 2008.

ROZAS, O. et al. Organic micropollutants (OMPs) in natural waters: Oxidation by $\mathrm{UV} / \mathrm{H}_{2} \mathrm{O}_{2}$ treatment and toxicity assessment. Water Research. v. 98, p.109118, 2016.
SOBRERO, M. C.; RONCO, A. Ensayo de toxicidad aguda con semillas de lechuga (Lactuca sativa L.). In: G. C. Morales (ed.), Ensayos Toxicológicos y Métodos de Evaluación de Calidad de Agua: Estandarización, Intercalibración, Resultados y Aplicaciones, IMTA, México, 2004.

SOUZA, D. R. et al. Degradação Fotocatalítica de Corante Alimentício Azorrubina e Monitoramento de Redução de Toxicidade. Orbital - The Electronic Journal of Chemistry, North America, v. 1, jan. 2016.

SUBTIL, E. L.; MIERZWA, J. C.; HESPANHOL, I. Avaliação do desempenho do sistema $\mathrm{UV} / \mathrm{H}_{2} \mathrm{O}_{2}$ no tratamento de efluentes provenientes do processo de tratamento térmico de emulsões de água e óleo. Rev. Ambient. Água, Taubaté, v. 4, n. 3, p. 169-180, 2009.

WILL, I. B. S. et al. Photo-Fenton degradation of wastewater containing organic compounds in solar reactors. Sep. Purif. Technol., v. 34, p. 51-57, 2004. 